\title{
Marcum Q-function as an analytical solution for misaligned Gaussian beams (Erratum)
}

\section{Claisso P. Azzolin, Airton F. Gurgel Jr., and Vítor Gouvêa Andrezo Carneiro*}

Military Institute of Engineering, Electrical Engineering Department, Photonics Laboratory, Rio de Janeiro, Brazil

[DOI: 10.1117/1.OE.60.7.079801]

This article [Opt. Eng. 60 (5), 056105 (2021) DOI: 10.1117/1.OE.60.5.056105] was originally published on May 24, 2021, with a normalization error in Fig. (3), which showed measurements of received power rather than attenuation.

The original figure was:

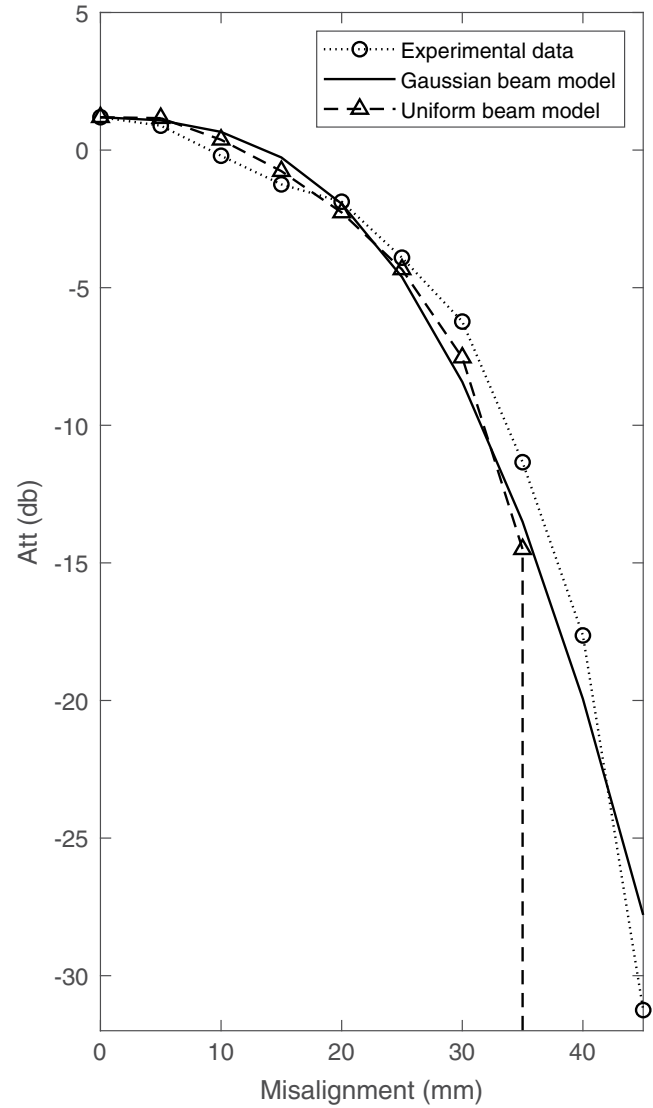

(a)

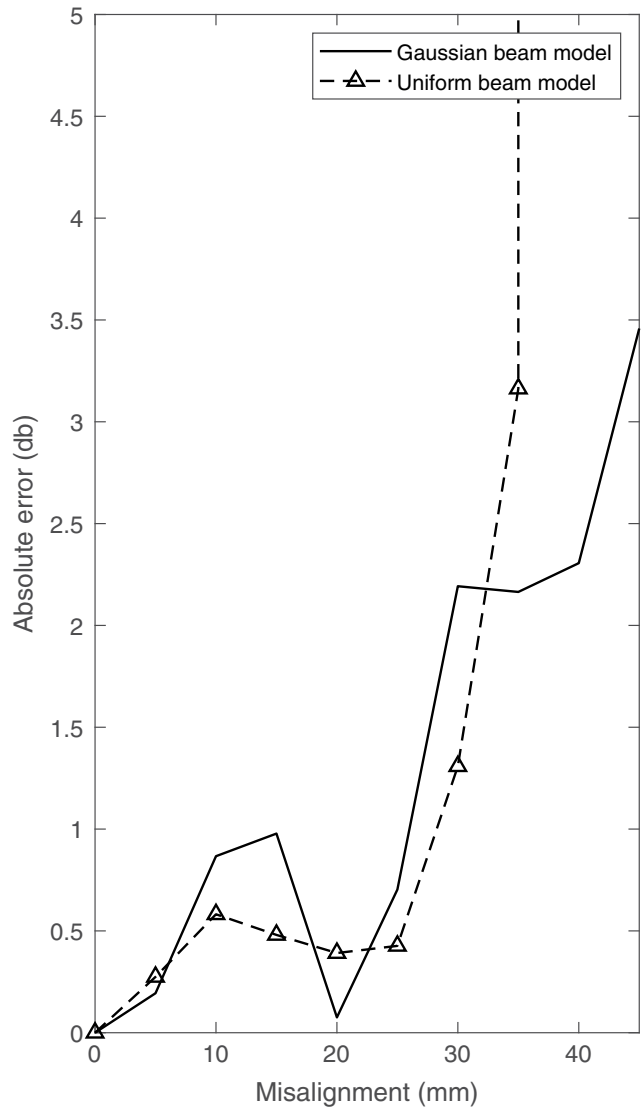

(b)

Fig. 3 Experimental and theoretical model for Gaussian beam misalignment. (a) Experimental and theoretical model for geometric and misalignment attenuation. (b) Absolute attenuation error.

*Address all correspondence to Vítor Gouvêa Andrezo Carneiro, andrezo@ime.eb.br 
The corrected figure is:

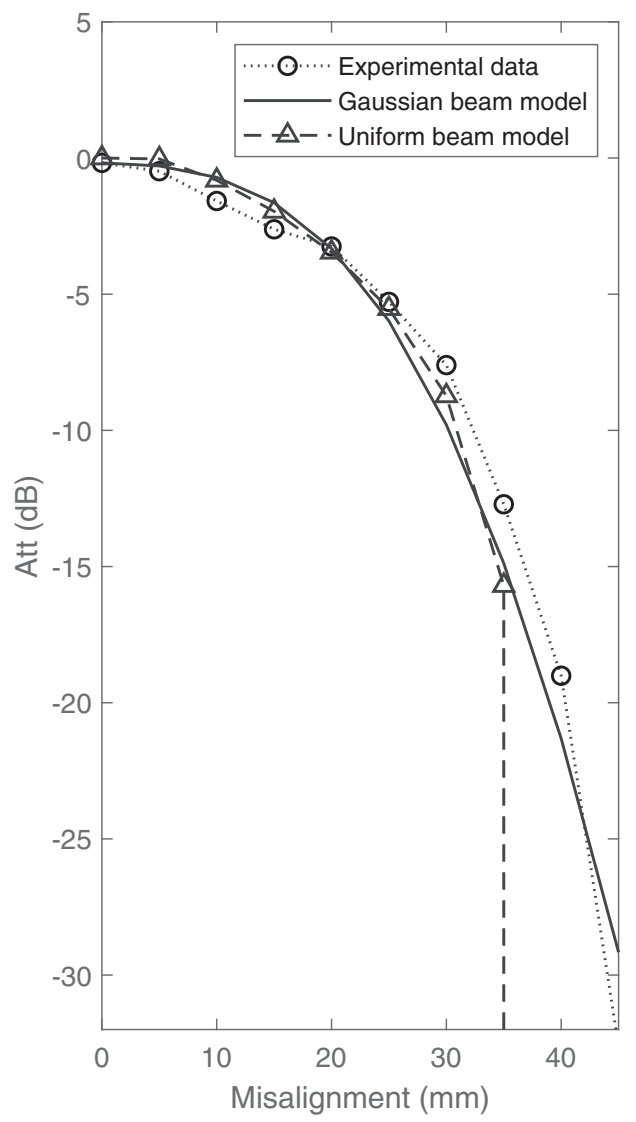

(a)

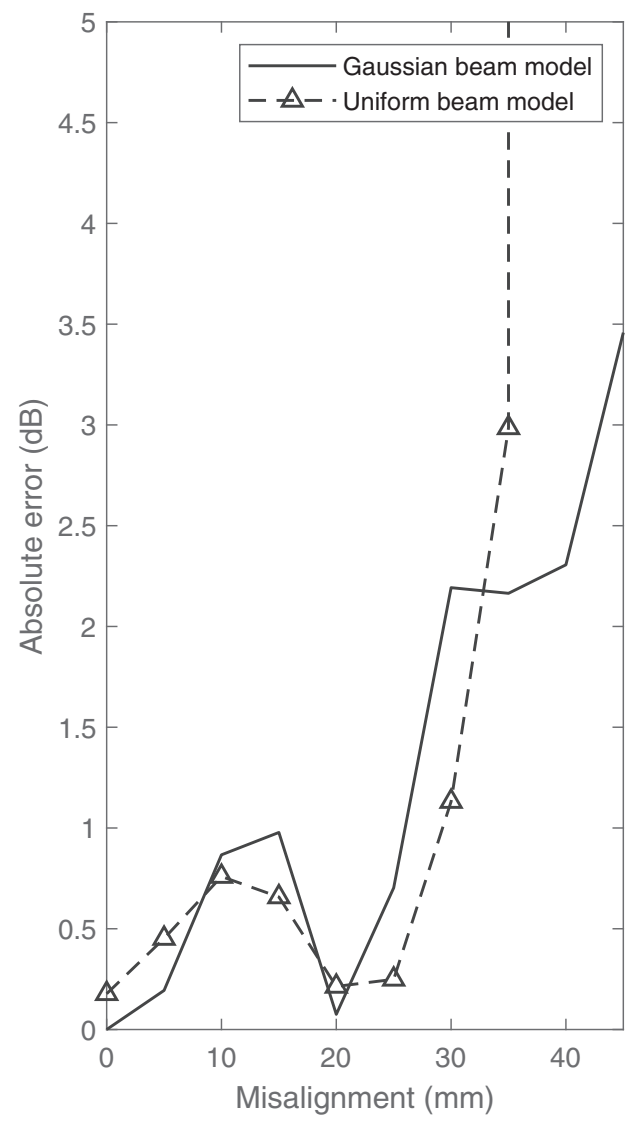

(b)

Fig. 3 Experimental and theoretical model for Gaussian beam misalignment. (a) Experimental and theoretical model for geometric and misalignment attenuation. (b) Absolute attenuation error.

The paper was corrected on 21 July 2021. 